\title{
Influence of State of Charge estimation uncertainty on energy management strategies for Hybrid Electric Vehicles
}

\author{
V. Larsson * L. Johannesson ${ }^{* *}$ B. Egardt ${ }^{* * *}$ \\ *e-mail: viktor.n.larsson@chalmers.se \\ **e-mail: larsjo@chalmers.se \\ ***e-mail: bo.egardt@chalmers.se \\ Chalmers University of Technology, Göteborg, Sweden
}

\begin{abstract}
:
This paper studies how the optimal energy management of a hybrid electric vehicle and a plug-in hybrid electric vehicle is affected by uncertain estimates of the battery state of charge. A simple model for the battery dynamics and the state of charge estimation is postulated, inspired by the known characteristics of previously proposed estimation schemes. Based on the assumption that the drive cycle is perfectly known, the effects of state of charge estimation uncertainty is studied by including the estimation uncertainty in the optimization of the energy management strategy. The simulations indicate lower battery usage and higher fuel consumption as the estimation uncertainty increases.
\end{abstract}

Keywords: Hybrid vehicles, Dynamic Programming, Stochastic control

\section{INTRODUCTION}

Increased electrification of powertrains has during the last decade emerged as a promising technology for reducing the environmental impact of road transportation. The first electrified vehicles introduced to the mass market were Hybrid Electric Vehicles (HEVs), which in addition to the Internal Combustion Engine (ICE) are equipped with an Electric Machine (EM) and an energy buffer. The $\mathrm{HEV}$ powertrain improves the energy efficiency by engine downsizing and brake energy recovery.

From a control engineering perspective the additional traction source in the HEV powertrain introduces a degree of freedom in meeting the driver's traction request. During recent years significant attention has been given to the development of control strategies, also called Energy Management Strategies (EMS), that exploit this degree of freedom in an efficient way, see Paganelli et al. (2002), Musardo et al. (2005) and Sciarretta and Guzzella (2007). Common to all proposed EMS are that they aim to minimize fuel consumption and/or emissions, while satisfying constraints on the battery charge level in order to maintain battery life length. The key variable to control is therefore the battery State of Charge (SoC), defined as the remaining battery capacity divided with the nominal battery capacity.

However, a complicating fact when designing the EMS is that SoC is a non measurable quantity that is dependent on the ionic concentrations at the anode/cathode. When implementing the EMS the SoC must therefore be estimated by the Battery Management System (BMS). The

\footnotetext{
‡ This work has been supported by the Swedish Hybrid Vehicle Centre (SHC).
}

estimation is typically based on a sensor fusion of current and voltage measurements, where the measured current is integrated and the inherent drift is compensated for by comparing the measured battery terminal voltage with the predicted voltage from a dynamic battery model. Several different estimation techniques have been suggested for the BMS; one is to use an Extended Kalman Filter as proposed by Plett (2004), another is to use Particle Filtering and Bayesian estimation, see Saha et al. (2007). However, no matter what estimation technique is used, there will always be a relatively large SoC estimation uncertainty due to the difficulty of accurately modeling the battery terminal voltage during dynamic operation.

All published methods known to the authors for designing an EMS are based on the certainty equivalence principle (see any text book in optimal control e.g. Anderson and Moore (1971)), meaning that the EMS is optimized based on the assumption that the SoC is perfectly known. The question in this paper is to investigate the validity of assuming certainty equivalence when designing an EMS for an HEV with a Li-Ion battery. The methodology used to answer this question is to first postulate a simple model for the SoC estimation uncertainty based on known characteristics of published SoC estimation schemes for Li-Ion batteries, see Plett (2004). The SoC estimation uncertainty model is then included in the optimization of the EMS and the resulting optimal EMS for a predetermined drive cycle is analyzed for varying levels of the estimation uncertainty. This is done in order to get an understanding of how uncertain SoC estimates affect the optimal EMS. Moreover, the study is done for both an HEV and a Plug-in HEV (PHEV) since these differ considerably in battery usage and depth of discharge. 


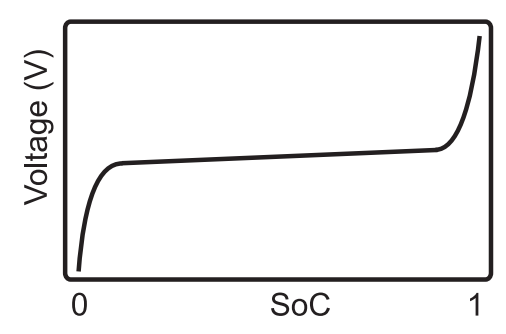

Fig. 1. Voltage vs. SoC for a typical Li-Ion battery.

\subsection{Paper Outline}

The outline of the paper is as follows. In Section 2 SoC estimation is explained briefly and the model of the $\mathrm{SoC}$ estimation uncertainty is postulated. Section 3 describes the vehicle model used in the paper and Section 4 covers the optimization of the EMS. Simulations are shown in Section 5 and the paper is ended with conclusions and discussion in Section 6.

\section{SOC ESTIMATION}

One can argue that the main problem with SoC estimation for Li-Ion batteries is not necessarily inaccurate current and voltage measurements, but rather that during dynamic operation the battery terminal voltage is only to a limited extent dependent on the SoC compared to other factors such as battery temperature and the memory and hysteresis effects. Hence, for precise SoC estimation a dynamic model for the battery terminal voltage is needed.

In order to accurately model the battery terminal voltage, a chemical model with several dynamic states and parameters is needed. However, such a model is typically not practical for implementation in a BMS, both due to the difficulty of obtaining correct values for the model parameters as well as the computational requirements. The battery is therefore normally modeled as an equivalent circuit consisting of a voltage source with one or several capacitors and resistors, see for example Musardo et al. (2005) and Sciarretta and Guzzella (2007). The open source voltage is often considered to depend on SoC according to a look-uptable determined by steady state voltage measurements at varying SoC levels. A typical voltage vs. SoC curve for a Li-Ion battery is shown in Figure 1. Furthermore, battery temperature and other dynamic effects such as memory and hysteresis effects are typically neglected.

In Figure 1 it is seen that the voltage vs. SoC curve is almost flat at intermediate SoC levels, meaning that in this region information obtained from voltage measurements will be of limited value. The effect is that the $\mathrm{SoC}$ estimator essentially relies on current integration at intermediate SoC levels and voltage measurements contribute by bringing down the uncertainty at low/high SoC values.

\subsection{Estimation Uncertainty Model}

In this section the behavior of the SoC estimator will be modeled with the purpose of capturing the main contributing phenomena that influence the estimation uncertainty, see Plett (2004).

The following model characteristics are postulated:
- the estimation uncertainty increases when the battery is used

- the estimation uncertainty decreases when the battery is not used

- the estimation uncertainty decreases at high and low SoC values

- the estimation uncertainty is independent of the battery temperature

- the SoC estimate is unbiased

The assumption of temperature independence is introduced since a thermal model of the battery is beyond the scope of this study. Furthermore, the SoC estimate is assumed to be unbiased to reduce the number of model parameters.

The estimation uncertainty model is based on a SoC model, a measurement model and a state dependent gain.

The discrete time SoC model is simply

$$
S o C_{k+1}=S o C_{k}+\frac{h i_{k}}{Q}+d_{k},
$$

and the discrete time measurement model is

$$
\mathrm{So}_{\mathrm{o}}=\mathrm{SoC}_{k}+w_{k} \text {. }
$$

Here $S o C$ represents the true state and $S \tilde{S} C$ corresponds to the "measured" state, i.e. the voltage measurement translated to SoC using the voltage vs. SoC curve. The sampling time is denoted $h, Q$ is the nominal capacity of the battery and $i$ is battery current and control signal to the system.

The distributions of the disturbances $d$ and $w$ are given by

$$
\begin{aligned}
d_{k} & \sim \mathbb{N}\left(0, \gamma+\beta\left|i_{k}\right|\right), \\
w_{k} & \sim \mathbb{N}\left(0, \mu \cdot \alpha\left(S o C_{k}\right)\right) .
\end{aligned}
$$

The variance of $d$ increases with current, thus representing that the SoC uncertainty will increase during dynamic operation both due to imprecise current measurements and modeling errors. The measurement variance, $w$, depends on the true state according to the function $\alpha(S o C)$, representing the inverse of $d V / d S o C$ as shown in Figure 2 , capturing that the voltage measurements give more information at low/high SoC values. The parameters $\gamma$, $\beta$ and $\mu$ are tuned to represent different estimator and battery characteristics.

The estimated state, $\hat{S o C}$, is determined using a state dependent gain, $K_{k}=K\left(\hat{S o} C_{k}, p_{k}\right)$, and is given by

$$
\hat{S o} C_{k+1}=\hat{S o C_{k}}+\frac{h i_{k}}{Q}+K_{k}\left[\hat{S} \tilde{o} C_{k}-\hat{S o C_{k}}\right]
$$

where $p$ represents the estimation uncertainty, i.e. the variance of the estimation error, $p=\mathbb{E}\left[e^{2}\right]$.

The estimation error becomes

$$
\begin{aligned}
e_{k+1} & =S o C_{k+1}-S \hat{o} C_{k+1} \\
& =\left(1-K_{k}\right) e_{k}+d_{k}-K_{k} w_{k} .
\end{aligned}
$$

Using basic calculus it is straightforward to show that

$$
\begin{aligned}
p_{k+1} & =\left(1-K_{k}\right)^{2} p_{k}+\sigma_{d, k}^{2}+K_{k}^{2} \sigma_{w, k}^{2}, \\
& =p_{k}+\left(p_{k}+\sigma_{w, k}^{2}\right)\left[K_{k}-\frac{p_{k}}{p_{k}+\sigma_{w, k}^{2}}\right]^{2} \\
& -\frac{p_{k}^{2}}{p_{k}+\sigma_{w, k}^{2}}+\sigma_{d, k}^{2},
\end{aligned}
$$


where $\sigma_{d}^{2}=\mathbb{E}\left[d^{2}\right]=\gamma+\beta|i|$ and $\sigma_{w}^{2}=\mathbb{E}\left[w^{2}\right]=\mu \cdot \alpha(S o C)$.

Equation (7) implies that the estimation uncertainty is minimized if the state dependent gain is chosen as

$$
K_{k}=\frac{p_{k}}{p_{k}+\sigma_{w, k}^{2}} .
$$

Finally with the gain chosen according to Equation (8) the estimation uncertainty becomes

$$
p_{k+1}=\frac{p_{k} \sigma_{w, k}^{2}}{p_{k}+\sigma_{w, k}^{2}}+\sigma_{d, k}^{2} .
$$

However, since the true state is not measurable, the exact value of $\sigma_{w}^{2}$ is unknown. Rather than assuming that $S o C \approx$ $\hat{S o C}$, a better approximation should be achieved if all the information of the current state estimate is used. Hence the true state is assumed to be $\beta$-distributed around the estimated state as $S o C \sim \mathbb{B}(\hat{S o C}, p)$, meaning that $\sigma_{w}^{2}$ can be calculated as

$$
\begin{aligned}
\sigma_{w}^{2} \approx \hat{\sigma}_{w}^{2} & =\mathbb{E}\{\mu \cdot \alpha(S o C) \mid \hat{S o C}\} \\
& =\int_{0}^{1} \mu \cdot \alpha(S o C) \beta(S o C, a, b) d S o C,
\end{aligned}
$$

where $\beta(S o C, a, b)$ is the probability density function of the $\beta$-distribution, given by

$$
\beta(S o C, a, b)=\frac{S o C^{a-1}(1-S o C)^{b-1}}{\int_{0}^{1} S o C^{a-1}(1-S o C)^{b-1} d S o C} .
$$

The shaping parameters $a$ and $b$ are determined by the mean and varience of the $\beta$-distribution and are thus given by

and

$$
a=\hat{S o C}\left(\hat{S o C}(1-\hat{S o C}) p^{-1}-1\right)
$$

$$
b=(1-\hat{S o} C)\left(\hat{S o} C(1-\hat{S o C}) p^{-1}-1\right) .
$$

The characteristics of the estimation uncertainty model are illustrated in Figure 2, where Equation (9) is simulated forwards in time for various values of the tuning parameters $\gamma, \beta$ and $\mu$ at constant values of the SoC and the current. The figure shows that the steady state estimation uncertainty increases with increasing values of $\gamma, \beta$ and $\mu$. Furthermore, intermediate $\mathrm{SoC}$ values and increasing currents results in a higher uncertainty. The transient response in the lower left plot illustrates the effect of zero battery current.

\section{VEHICLE MODELING}

Two vehicle concepts are modeled in this paper, one HEV and one PHEV. Both concepts have a parallel configuration with the electric machine placed directly at the drive axis, see Figure 3, representing two different models built on the same vehicle platform. The differences between the concepts are related to the battery; the PHEV has a more powerful battery with higher capacity in order to provide some $25 \mathrm{~km}$ of electric driving. Furthermore, the two concepts have different constraints on the battery usage, the PHEV is allowed to use $65 \%$ of the battery capacity whereas the HEV is only allowed to use $20 \%$. These constraints are imposed on the battery usage due to battery ageing, which is strongly correlated with the
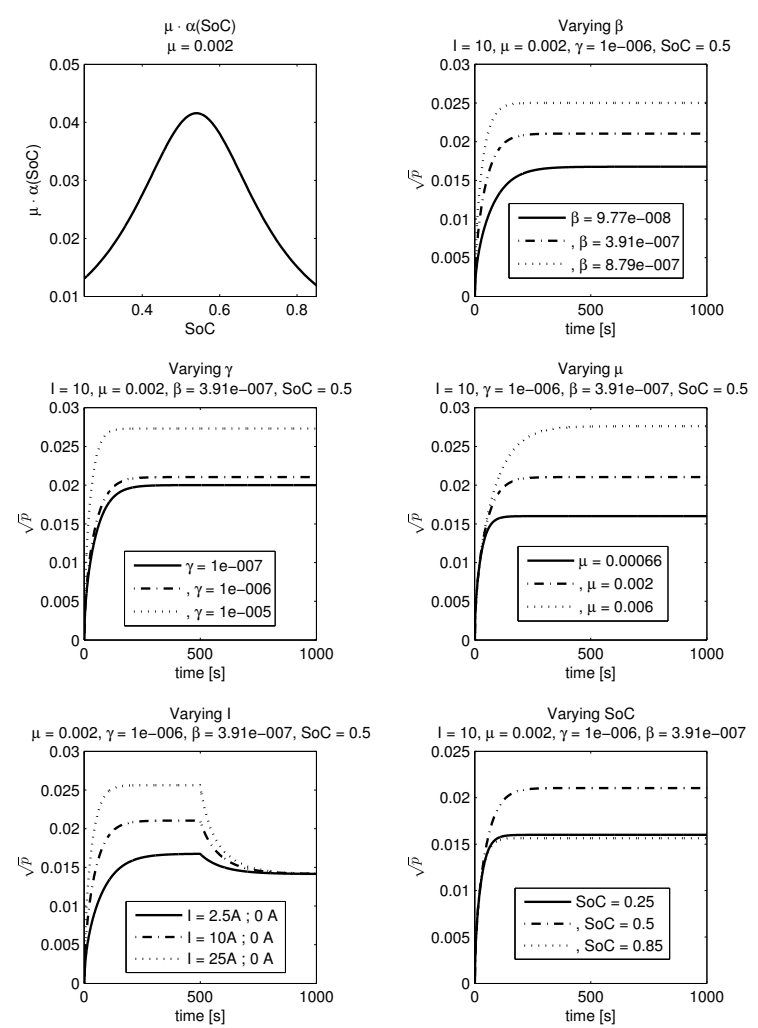

Fig. 2. The measurement variance $\mu \cdot \alpha($ SoC $)$, upper left plot, and the estimation uncertainty, $p$, for various values of the tuning parameters, the battery SoC and the battery current.

battery depth of discharge, see Vetter et al. (2005). The properties of the vehicle concepts are summarized in Table 1.

Table 1. Vehicle Model Data

\begin{tabular}{|l|l|l|}
\hline \multicolumn{2}{|c|}{ Vehicle Data } \\
\hline \multirow{2}{*}{ HEV } & Battery & Li-Ion $-192 \mathrm{~V}, R_{i n}=0.30 \Omega$ \\
& & $1.4 \mathrm{kWh}(0.28 \mathrm{usable})$ \\
& & $S o C_{0} \approx S o C_{f} \approx 0.65$ \\
\hline \multirow{2}{*}{ PHEV } & Battery & Li-Ion $-305 \mathrm{~V}, R_{i n}=0.13 \Omega$ \\
& & $8 \mathrm{kWh}(4.8 \mathrm{usable})$ \\
ICE & & $S o C_{0}=0.85, S o C_{f} \approx 0.25$ \\
\hline Chassis & & Spark Ignited - 50 kW \\
& Transmission & Permanent Magnet - 50 kW \\
& Gear ratios, $r_{g b, i}$ & $4.2,2.5,1.6,1.1,0.9$ \\
& Final drive, $r_{f d}$ & 4.2 \\
& Wheel radius, $r_{w h}$ & $0.3 \mathrm{~m}$ \\
& Mass, $m$ & $1500 \mathrm{~kg}$ \\
& Air resist., $c_{d} A_{f}$ & $0.66 \mathrm{~m}^{2}$ \\
& Rolling resist., $f_{r}$ & 0.012 \\
& Power elec. eff., $\eta_{p e}$ & 0.95 \\
& Gearbox eff., $\eta_{g b}$ & 0.97 \\
& Final drive eff., $\eta_{f d}$ & 0.95 \\
\hline
\end{tabular}

\subsection{Powertrain Model}

The powertrain is modeled using a quasi static approach, meaning that the ICE mass fuel rate and the EM losses are determined through linear interpolation between steady state measurements, neglecting the internal dynamics of the ICE and the EM. The gearbox, the final drive and the 




Fig. 3. The HEV/PHEV configuration.

power electronics are assumed to have constant efficiencies. The battery is modeled as a voltage source in series with an internal resistance, $R_{i n}$, the open circuit voltage is dependent on SoC, as in Figure 1, and the internal resistance is assumed to be constant over the SoC interval of normal usage. Dynamic effects such as hysteresis, memory and battery temperature are neglected.

The torque demand at the drive axis, $\tau_{d e m}$, needed to follow a predetermined drive cycle is calculated using an inverse simulation approach, see Guzzella and Sciarretta (2007), is

$\tau_{d e m}=\frac{r_{w h}}{r_{f d}}\left(\frac{\rho_{a i r}}{2} A_{f} c_{d} v^{2}+f_{r} m g \cos \theta+m g \sin \theta+m \frac{d v}{d t}\right)$,

where $v$ and $\theta$ represents the drive cycle velocity and road grade. The interesting equation from a control engineering perspective is the torque split between the ICE and the EM, given by

$$
\tau_{d e m}+\tau_{f r}=\left(\tau_{e m} r_{f d}+\tau_{i c e} r_{f d} r_{g b, i} \eta_{g b}\right) \eta_{f d},
$$

where $\tau_{f r}$ is the torque applied by the mechanical brakes. The choice of gear, $r_{g b, i}$, is given by a predetermined gear shift strategy, i.e. a look up table as a function of the vehicle speed with an additional hysteresis to avoid excessive gear shifting. The dynamics during the gearshift are neglected.

\section{OPTIMAL ENERGY MANAGEMENT STRATEGIES}

If the drive cycle is considered to be deterministic the optimal EMS can be determined by solving the following optimization problem

$$
\begin{gathered}
J^{*}=\min _{\left\{u_{k}\right\}} \underset{\left\{d_{k}, w_{k}\right\}}{\mathbb{E}}\left\{\Upsilon\left(x_{N}\right)+\sum_{k=1}^{N-1}\left(\dot{m}_{k}\left(u_{k}\right)+\Gamma\left(x_{k}\right)\right)\right\} \\
\text { s.t. } x_{k+1}=f\left(x_{k}, u_{k}, z_{k}\right) \\
\quad x_{k} \in X, u_{k} \in U\left(z_{k}, x_{k}\right), z_{k} \in Z
\end{gathered}
$$

where

$$
\begin{aligned}
& x_{k}=\left[S o C_{k}, \hat{S o} C_{k}, p_{k}, I C E_{s, k}\right]^{T} \text {, } \\
& y_{k}=\left[\hat{S o} C_{k}, p_{k}, I C E_{s, k}\right]^{T} \text {, } \\
& u_{k}=\left[\tau_{i c e, k}, I C E_{d, k}\right]^{T} \text {, } \\
& z_{k}=\left[d_{k}, w_{k}, v_{k}, \theta_{k}, r_{g b, k}\right]^{T} \text {. }
\end{aligned}
$$

Here the state vector $x$, consists of the the true SoC, the SoC estimate $\hat{S o C}$, the variance of the estimate $p$, and the
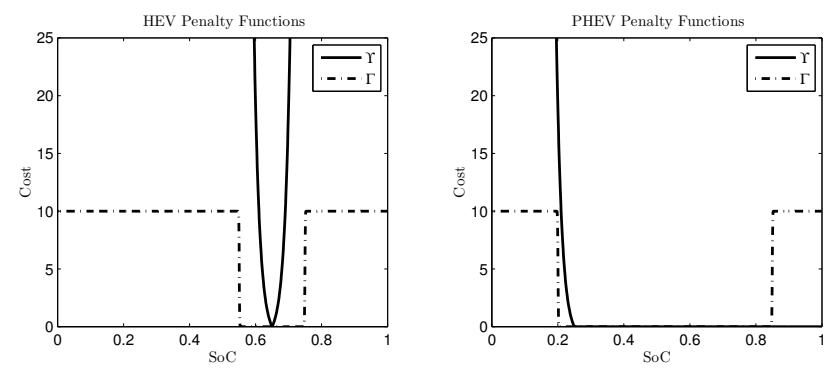

Fig. 4. The penalty functions $\Upsilon$ and $\Gamma$ plotted vs true SoC for the $\mathrm{HEV}$ and PHEV concepts.

engine state $I C E_{s}$. The measurement vector $y$, consists of the state variables observable by the EMS. The control signal vector $u$, to the system consists of the ICE torque $\tau_{i c e}$, (the EM torque $\tau_{e m}$, is given implicitly by the torque split) and the decision to change ICE state $I C E_{d}$. Finally, the disturbance vector $z$, consists of the stochastic SoC process disturbance $d$, the stochastic SoC measurement disturbance $w$ and the deterministic drive cycle, i.e. the velocity profile $v$, the road grade $\theta$ and the gear $r_{g b}$, (given by the gear shift strategy). Furthermore, the variables in the control signal vector, state vector and disturbance vector are constrained to be within the physical limitations of the powertrain model in Section 3.1.

The performance index, $J$, is composed of the fuel mass rate, $\dot{m}$, and $\Gamma$ which is a penalty function ensuring that the SoC is kept approximately within allowed bounds. Finally the end cost, $\Upsilon$, penalizes deviations from the preferred SoC value at the end of the driving mission, $\mathrm{SoC}_{f}$.

The optimization problem described by Equations (16)(17) is solved using Dynamic Programming (DP), see Bellman and Dreyfus (1962) or Bertsekas (2000).

The DP formulation of the problem becomes $J_{k}\left(Y_{k}\right)=\min _{u_{k}} \underset{d_{k}, w_{k}}{\mathbb{E}}\left\{\dot{m}_{k}\left(u_{k}\right)+\Gamma\left(Y_{k}\right)+J_{k+1}\left(Y_{k+1}\right) \mid Y_{k}\right\}$,

where the cost-to-go function, $J_{k}$ is determined over the discretized part of the observable state space, $Y_{k}$. The cost-to-go function is iterated recursively backwards in time from the final time sample $\mathrm{N}$ to first, using linear interpolation. At the final time sample the cost function is initialized as $J_{N}\left(Y_{N}\right)=\Upsilon\left(Y_{N}\right)$.

The optimal EMS is then determined by iterating Equation (18) forwards in time according to

$$
u_{k}^{*}=\arg \min _{u_{k}} \underset{d_{k}, w_{k}}{\mathbb{E}}\left\{\dot{m}_{k}\left(u_{k}\right)+\Gamma\left(y_{k}\right)+J_{k+1}\left(y_{k+1}\right) \mid y_{k}\right\} \text {. }
$$

Note that the penalty functions, $\Upsilon$ and $\Gamma$ shown in Figure 4 , are defined to penalize undesired true SoC values. This means that when optimizing the EMS, the penalty will be smoothed out since $\Upsilon$ and $\Gamma$ are determined by taking the expectation over the true SoC distribution, given by Equation (11).

\section{SIMULATIONS}

Simulations were performed both for the HEV and PHEV vehicle concepts on the drive cycle shown in Figure 5. The drive cycle consists of the HWFET and FTP72 test cycles, 
combined with a plausible altitude profile to make the driving conditions more realistic.

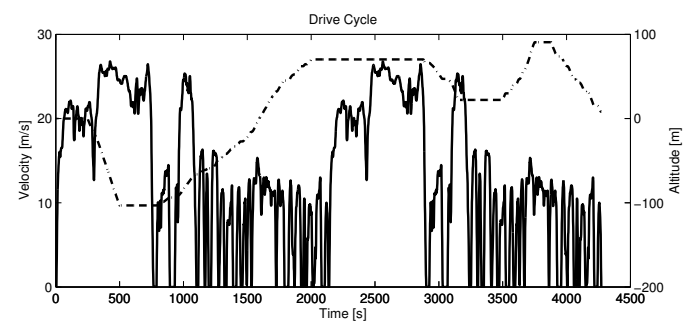

Fig. 5. The drive cycle used during the simulations.

Using the estimation uncertainty model and the powertrain model, outlined in Sections 2.1 and 3.1, optimal EMS were determined by solving the DP formulation of the optimization problem, given by Equation (18) in Section 4. The optimal sequence of control actions was determined by iterating Equation (19) forwards in time while generating a realization of the stochastic processes $d$ and $w$ using a pseudorandom number generator.

To illustrate the sensitivity of the optimal EMS against variations in the average estimation uncertainty the $\mu$ parameter was varied between a low, a medium and a high value, while $\gamma$ and $\beta$ were kept constant at; $\beta=3.9 \cdot 10^{-7}$, $\gamma=1 \cdot 10^{-6}$. For each $\mu$-value the average value of the final SoC estimate and the fuel consumption were calculated (neglecting the cost to recharge the PHEV battery). The results based on 50 realizations are shown in Table 2.

The optimal EMS of the HEV and PHEV concepts, for a few realizations, are shown in Figures 6-9. Figures 6 and 8 correspond to the medium $\mu$-value and show in descending order; the true $\mathrm{SoC}$ and the estimated SoC, the estimation error, the approximate estimation uncertainty (i.e. $\sqrt{p}$ given by Equation (9) using the approximation in Equation (10)) and the battery current. The figures 7 and 9 depicts the results for the low and high $\mu$-values, however here only the true SoC, the estimated SoC and the approximate estimation uncertainty are shown.

Table 2. Simulated average values of the fuel consumption and the final SoC estiamte.

\begin{tabular}{|c|c|c|c|c|c|c|}
\hline \multicolumn{8}{|c|}{ Simulation Data } \\
\hline & \multicolumn{3}{|c|}{ Final SoC est. $\left[S \hat{o} C_{f}\right]$} & \multicolumn{3}{c|}{ Fuel Cons. $[l \backslash 100 k m]$} \\
\cline { 2 - 7 } & \multicolumn{2}{|c|}{$\mu$-value $\left[10^{-3}\right]$} & \multicolumn{3}{|c|}{$\mu$-value $\left[10^{-3}\right]$} \\
\cline { 2 - 7 } Concept & $\begin{array}{c}\mu_{\text {low }} \\
=0.7\end{array}$ & $\begin{array}{c}\mu_{\text {med }} \\
=2.0\end{array}$ & $\begin{array}{c}\mu_{\text {high }} \\
=6.0\end{array}$ & $\begin{array}{c}\mu_{\text {low }} \\
=0.7\end{array}$ & $\begin{array}{c}\mu_{\text {med }} \\
=2.0\end{array}$ & $\begin{array}{c}\mu_{\text {high }} \\
=6.0\end{array}$ \\
\hline HEV & 0.655 & 0.658 & 0.659 & 3.49 & 3.51 & 3.55 \\
\hline PHEV & 0.281 & 0.284 & 0.296 & 1.16 & 1.18 & 1.23 \\
\hline
\end{tabular}

\subsection{HEV Concept}

The simulations for the HEV concept, shown in Figures 6 and 7, suggest that the estimation uncertainty remains almost constant throughout the drive cycle. This is a consequence of the $\mathrm{HEV}$ operating region close to $\mathrm{SoC}=$ 0.65 where voltage measurements give limited information, i.e. the SoC dependent measurement noise $w$ has a fairly constant and high variance. Also notable is that when the average estimation uncertainty increases the battery usage decreases, i.e. the SoC deviations from 0.65 decreases. The reason behind this is that the strategy tries to reduce the cost of the penalty function $\Gamma$, which is determined by both the SoC estimate and the estimation uncertainty. Moreover, the average fuel consumption, see Table 2, increases with higher uncertainty, an intuitive result since decreased battery usage limits the degree of freedom of the EMS.
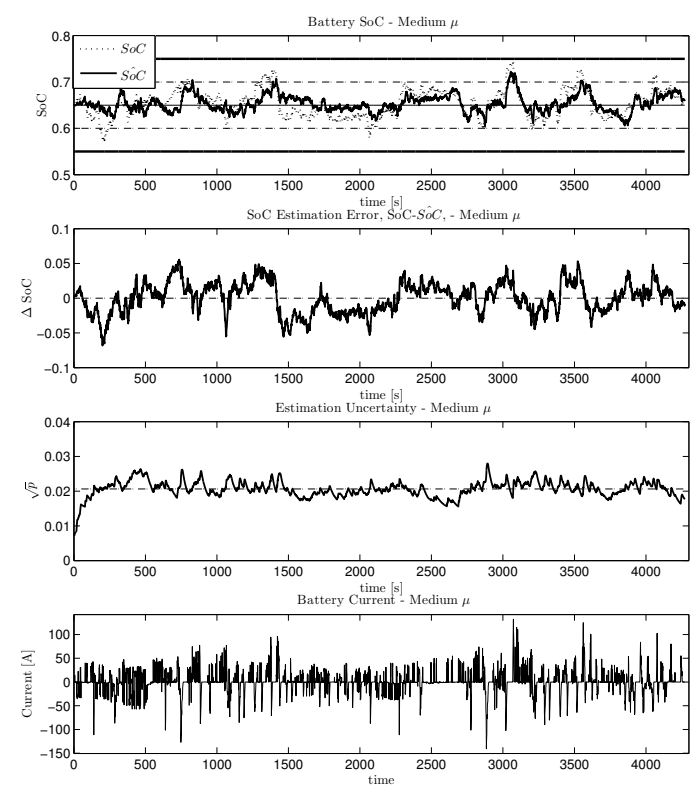

Fig. 6. Simulation of the HEV, medium $\mu$-value.
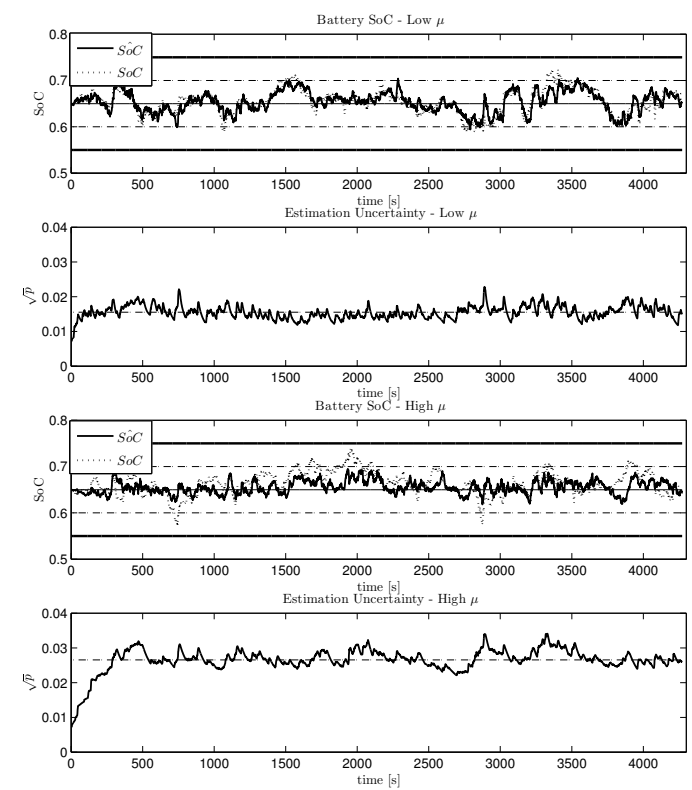

Fig. 7. Simulation of the HEV, low and high $\mu$-values.

\subsection{PHEV Concept}

Simulations for the PHEV concept, shown in Figures 8 and 9 , indicate that the estimation uncertainty varies more during a drive cycle with a PHEV than with an HEV. One reason is that the $\mathrm{SoC}$ changes more throughout a drive cycle for a PHEV, meaning that the variance of measurement noise, $w$, has a higher variation. Furthermore, the estimation uncertainty shows a tendency to decrease towards the end of the drive cycle. This is partly motivated by that 
the measurements give more information since the SoC is low, although the main reason is that the EMS reduces the battery current in order to end the drive cycle with a more precise SoC estimate and thus a lower end cost $\Upsilon$. Finally, also for the PHEV the average fuel consumption increases with increased estimation uncertainty, here explained by decreased battery usage at the end of the drive cycle and an increased final SoC for higher estimation uncertainties as seen in Table 2.


Fig. 8. Simulation of the PHEV, medium $\mu$-value.


Fig. 9. Simulation of the PHEV, low and high $\mu$-values.

\section{CONCLUSION AND DISCUSSION}

The simulation results indicate that uncertainties regarding the SoC estimate affect the optimal EMS of an HEV and a PHEV differently. For an HEV with steep/discontinuous penalty functions it is clear that increased estimation uncertainty leads to decreased battery usage and therefore higher fuel consumption. However, the magnitude of this effect will depend strongly on the SoC constraints; smoother penalty functions and increasing the allowed depth of discharge will decrease the effect.

The optimal EMS of a PHEV is not affected in the same manner since the constraints on the SoC usage are less restrictive in terms of the depth of discharge. Instead of decreased battery usage throughout the whole drive cycle, the trend is toward decreased battery usage during the final part and a higher final SoC estimate, this in order to receive a lower end cost. The effect is enhanced by steep/discontinuous penalty functions.

To summarize, the results suggest that the assumption of certainty equivalence when deriving the EMS is more appropriate for a $\mathrm{PHEV}$ than for an $\mathrm{HEV}$, at least with sharp constraints on the SoC usage.

Finally, it is worth to point out that the postulated model of the SoC dynamics, described by Equation (1), has the property that battery energy essentially can be created or vanish in a random walk behavior due to the $\mathrm{SoC}$ disturbance. Therefore the modeling approach used in this paper is not very well suited for studying energy efficiency figures of individual realizations.

\section{REFERENCES}

Anderson, B.D.O. and Moore, J.B. (1971). Linear Optimal Control. Prentice-Hall, Inc, Englewood Cliffs, N.J.

Bellman, R.E. and Dreyfus, S.E. (1962). Applied Dynamic Programming. Princeton University Press.

Bertsekas, D. (2000). Dynamic Programming and Optimal Control. Athena Scientific, Bellmont Massachusets.

Guzzella, L. and Sciarretta, A. (2007). Vehicle Propulsion Systems. Springer Verlag, Berlin Heidelberg, 2 edition.

Musardo, C., Rizzoni, G., Guezennec, Y., and Staccia, B. (2005). A-ECMS: An Adaptive Algorithm for Hybrid Electric Vehicle Energy Management. European Journal of Control, 11(4-5), 509-524.

Paganelli, G., Delprat, S., Guerra, T., Rimaux, J., and Santin, J. (2002). Equivalent consumption minimization strategy for parallel hybrid powertrains. Vehicular Technology Conference. IEEE 55th Vehicular Technology Conference. VTC Spring 2002, 4, 2076-2081.

Plett, G. (2004). Extended Kalman filtering for battery management systems of LiPB-based HEV battery packsPart 1-3. Journal of Power Sources, 134(2), 252292.

Saha, B., Poll, S., Goebel, K., and Christophersen, J. (2007). An integrated approach to battery health monitoring using bayesian regression and state estimation. 2007 IEEE Autotestcon, 646-653.

Sciarretta, A. and Guzzella, L. (2007). Control of Hybrid Electric Vehicles. IEEE Control Systems Magazine, 6070 .

Vetter, J., Nov, P., Wagner, M.R., Veit, C., and Besenhard, J.O. (2005). Ageing mechanisms in lithium-ion batteries. Journal of Power Sources, 147, 269-281. 